\title{
Serendipitous UV source catalogues for 10 years of XMM and 5 years of Swift
}

\author{
V.N. Yershov
}

Received: 15 January 2014 / Accepted: 21 April 2014 / Published online: 1 May 2014

(C) The Author(s) 2014. This article is published with open access at Springerlink.com

\begin{abstract}
Two UV serendipitous source catalogues are presented which were compiled based on the observations with two similar UV telescopes, one being on-board the ESA's XMM Newton observatory and another-on-board the NASA's Swift satellite. Both telescopes have similar optical and registration systems providing photometry in three ultraviolet and three visible bands. After processing a 10 years long series of observations from XMM and 5 years from Swift, we have compiled two source catalogues containing more than 4 million sources for XMM and 6 million sources for Swift. We describe the processing algorithms and present catalogue characteristics in comparison with each other.
\end{abstract}

Keywords UV source catalogues - Statistics $\cdot$ XMM Newton · Swift

\section{Introduction}

The Swift Ultraviolet and Optical Telescope (UVOT) (Roming et al. 2005) and XMM Newton Optical Monitor (OM) (Mason et al. 2001) are relatively small $(0.3 \mathrm{~m})$ Ritchey-Chretien ultraviolet telescopes that provide photometry in three near-ultraviolet (UVW2, UVM2 and UVW1) and three visible (U, B, V) spectral bands. The telescope photodetectors use microchannel plate photomultipli-

V.N. Yershov ( $\varangle)$

Mullard Space Science Laboratory, University College London, Holmbury St. Mary, Dorking RH5 6NT, UK

e-mail: v.yershov@ucl.ac.uk

V.N. Yershov

Pulkovo Observatory, Pulkovskoye shosse 65, Saint Petersburg, 196140, Russia ers coupled to CCDs which allows single-event detections within the $17^{\prime}$ by $17^{\prime}$ fields of view. The XMM Newton mission was launched by ESA on 10th of December 1999, and Swift was launched by NASA on 20th of November 2004. Since then, they made thousands of observations of different targets, each field including many serendipitous sources.

The distribution of 6604 XMM Newton observations on the sky is shown in the upper panel of Fig. 1, and the lower panel shows a similar distribution for 23428 Swift observations. It is seen that in the second case the observations cover the sky more uniformly, which corresponds to the more uniform distribution on the sky of gamma-ray bursts - the principal targets of Swift.

The purpose of this work is to get photometric parameters of these sources by processing the series of observations made by the OM and UVOT telescopes, applying the appropriate instrument calibrations and compiling the catalogues of these parameters.

We have used a 5 years long series of observations from Swift and 10 years from XMM and compiled two catalogues of these sources. Both telescopes have similar registration systems, but during the manufacturing of the UVOT detecting system, it was achieved a higher sensitivity for the filters UVM2 and UVW2 compared to the same filters of the OM (see the plots in Fig. 5). Due to that, UVOT can detect more UV sources than OM.

\section{Data processing}

There are different stages (engines) of data processing, each stage achieving a certain intermediate result. For example, the first engine is designed for preparing raw images for further processing and also for creating the quality maps used at a later processing stage for assigning quality flags to those 

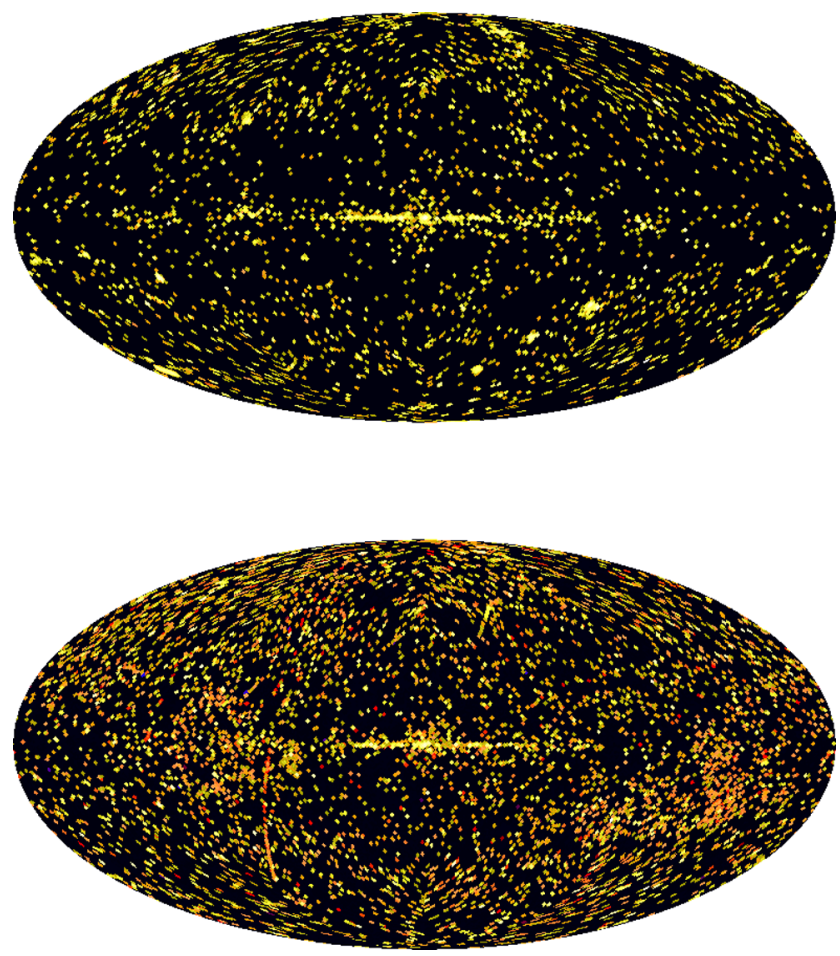

Fig. 1 Distribution of the OM (above) and UVOT (below) targets across the sky (lighter colours correspond to higher accumulated exposures)

sources whose photometry might be affected by image artefacts. The sequence of the OM and UVOT data processing is approximately the same for both telescopes. The main difference consists in that the source detection in the XMM $\mathrm{OM}$ processing pipeline is implemented on raw images (individual exposures), whereas the Swift UVOT images are first stacked together for each observation and only after that all of the serendipitous field sources are detected and parameterised by using these stacked images. The second algorithm has an advantage in that it allows reaching fainter sources, but we used the standard XMM SAS procedure to avoid unforeseen processing complications. Eventually, the detection of fainter sources on stacked OM images is implemented at the end of the OM processing chain, and these faint sources are then added to the source list of the $\mathrm{OM}$ catalogue.

Specialised software packages exist for processing both instruments data: these are the XMM Newton "Science Analysis System" (SAS) and Swift packages from NASA's High Energy Astrophysics Software (Heasoft) available at http://heasarc.gsfc.nasa.gov. These software packages are different although the OM and UVOT images and the processing algorithms are similar. The OM software packages are part of the SAS software, which is regularly updated and improved. For our purposes we have used the standard release SAS-13.0.1, in which we have improved some of the
OM packages based on the test data processing. We have processed the UVOT data by using the standard packages form Heasoft- 6.11 but we customised some the UVOT packages in order to get more complete source detection and to properly apply quality flags to those sources that were detected within the UVOT image artefacts.

The UVOT processing scheme is shown in Fig. 2 (the OM processing scheme is not shown here as it is similar). It consists of a few engines (scripts) calling the Swift tasks from the NASA Heasoft package.

The first engine deals mainly with image artefacts (readout streaks. scattered light features, etc.) creating a map of these artefacts and passing it to the further processing stages together with the preprocessed raw image. The preprocessing includes the image creation from the list of events (photon detections), unless the instrument was in the imaging mode of work, localises bad pixels (task uvotbadpix) and removes the modulo- 8 pattern caused by the specific centroiding algorithm implemented in the OM and UVOT detectors (task uvotmodmap). The task uvotflagqual identifies possible image artefacts and sets quality flags in the pixels of the quality map which accompanies the main image at the further processing steps and which is used at the final stage of processing for passing quality flags to those sources whose coordinates coincide with the coordinates of the flagged quality map pixels. An example of the quality map containing a readout streak, diffraction spikes and some other image artefacts is shown in Fig. 3, lower panel, which corresponds to a UVOT image shown in the upper panel. Checking the quality flag column when using the catalogues is highly advisable as this allows avoiding wrong conclusions about source photometry. Each flag is a binary number whose bits correspond to the presence or absence of an image artefact (the types of these artefacts will be given in the catalogue descriptions).

The second engine rotates the images with the purpose to align them along the celestial coordinate axes (task swiftxform). Then the script of the second engine corrects for the possible shifts of the image coordinates with respect to the sky coordinates by using reference stars from the USNO-B1 catalogue (task uvotaspcorr). At the end of this processing stage, the task uvotexpmap generate exposure maps corresponding to the sky-rotated and aspect-corrected images.

The third engine stacks different exposures for each filter in one observations, as well as the corresponding exposure and quality maps (task uvotimsum) and generates a largescale sensitivity map needed for accurate photometry (task uvotskylss).

The fourth engine calculates the background maps for each stacked image and detects sources whose count rates exceed 5-sigma threshold above the background (task uvotdetect). The task uvotdetect used for the catalogue processing was modified with respect to the standard code from the 
Fig. 2 Data processing engines for the UVOT catalogue

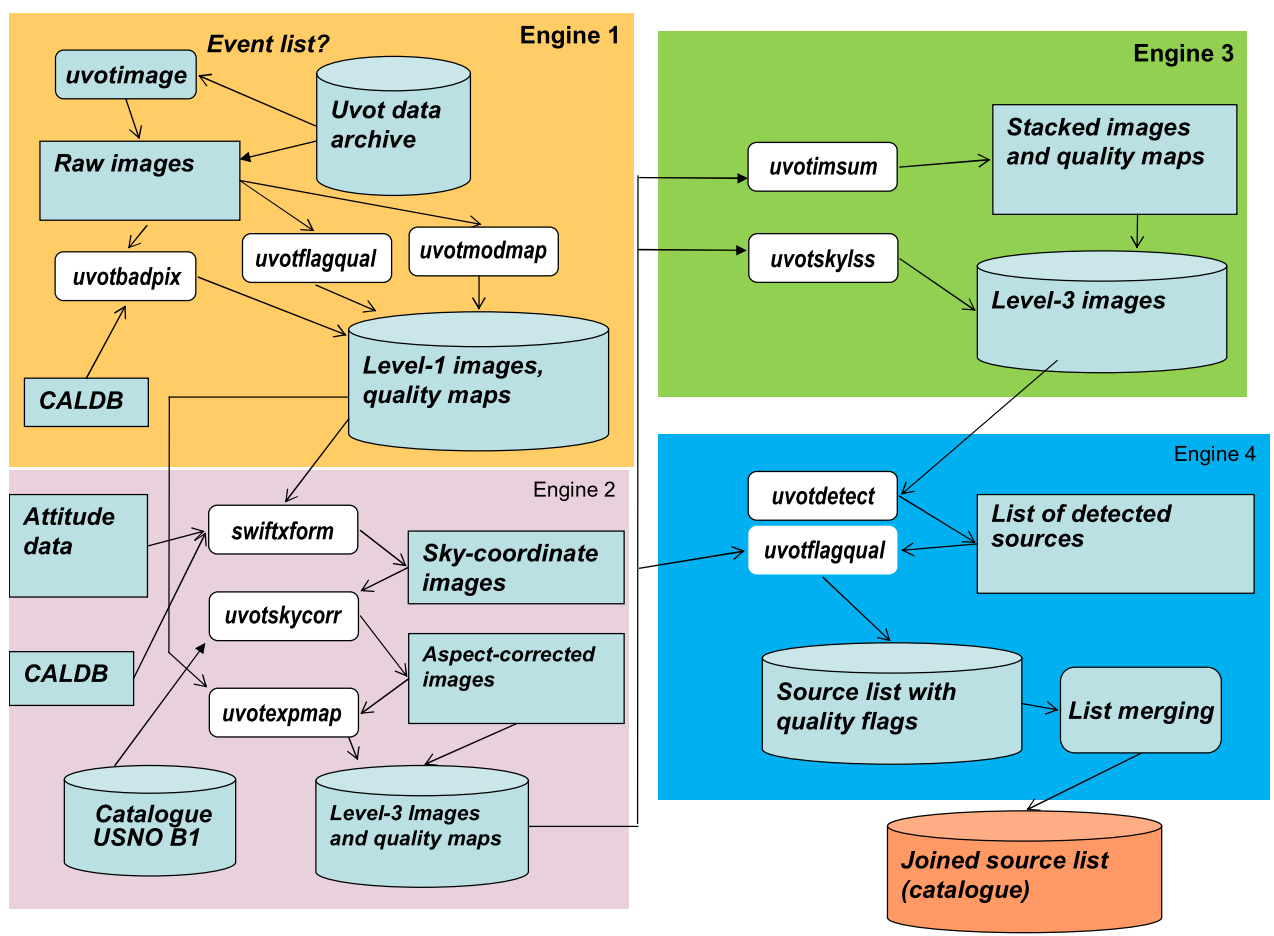

Heasoft package in order to deal with most of the problematic images that could be found in the UVOT archive. The final stage of the processing involves the task uvotflagqual that extracts the image quality flags from the quality maps produced by the first engine. This flags are introduced into the source lists for each observation. These source lists are eventually merged into the final source catalogue.

Since the images from different observations correspond to different source lists, the same source could be detected in different observations. Such sources have multiple entries in the final catalogue list, but they have the same unique source identification number, so the number of entries in the final source list table is larger than the total number of sources in the catalogue.

\section{Catalogue structure}

Both OM and UVOT source catalogues have similar structures. They are presented in the form of FITS files with two tables, one (called SOURCES) containing the source magnitudes and fluxes for 6 filters, as well as the magnitude and flux error estimates quality flags, etc., and the second table (SUMMARY) containing information about all of the observations used for producing the source catalogue (observation identification number, number of exposures, detection limits for each exposure, etc.). The first column (IAUNAME) of the sources table give the IAU source name in the form XMMOM JRRRRRR.R+DDDDDD for the OM catalogue and SWIFTUVOT JRRRRRR.R+DDDDDD for the UVOT cata- logue, where R stands for right ascension and D for declination coordinates. The second column (N_SUMMARY) containing a number linking the SOURCES and SUMMARY tables with each other, that is, for each source it gives the entry in the SUMMARY table corresponding to the observation in which the source was detected. More details about the catalogue data will be given in the description of the released versions of the catalogues.

Due to the differences in the UV-band effective areas the Swift UVOT catalogue contains an essentially larger number of sources detected for the filters UVW2 and UVM2 in the catalogue UVOTSSC compared to OM SUSS-2. The main features of these two catalogues are summarised in Table 1. The XMM OM catalogue is called SUSS-2, or the Serendipitous Ultraviolet Source Survey, the index " 2 " corresponding to the second data release (the first release was produced a few years ago to cover the period of XMM observations from 2000 to 2007; Page et al. 2012).

The Swift UVOT serendipitous source catalogue (UVOTSSC) is the first of this kind, the second (improved and extended) data release being planned for 2015 .

\section{Comparison of the catalogues}

Some of the sources in the UVOT and OM catalogues overlap. So the comparison of their magnitudes gives us a possibility to check the photometric accuracy of both catalogues. The result of this comparison is shown in Fig. 4 for three visible filters (upper panel) and three UV filters (lower panel). 

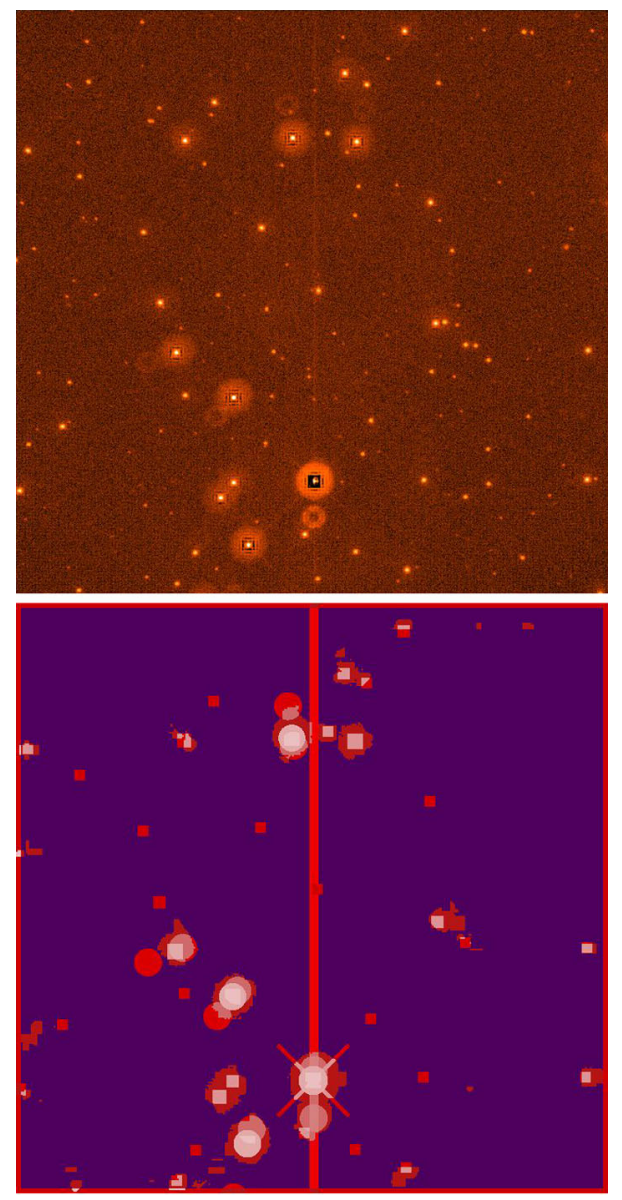

Fig. 3 Example of the UVOT image (above) with its corresponding quality map (below)

Table $1 \mathrm{OM}$ and UVOT source catalogue statistics

\begin{tabular}{lll}
\hline Catalogue & OM SUSS-2 & UVOTSSC \\
\hline Period of observations & $2000-2012$ & $2005-2010$ \\
Total observations & 6604 & 23428 \\
Total sources & 4008879 & 6270743 \\
Repeated observations & 692223 & 2052889 \\
Total entries & 5595331 & 14861238 \\
\hline
\end{tabular}

The visible filters of both instruments are almost identical, so the comparison plot shows one-to-one correspondence of the UVOT and OM magnitudes. It also shows the dependence of the source magnitude-error on magnitude. As for the UV filters (lower panel of Fig. 4), one can notice a systematic shift of about $\Delta m=0.75^{m}$ between the UVW1 magnitudes of OM and UVOT, which is slightly diminishing for larger source magnitudes as $\Delta m=0.75-0.07 \times$ $(m-15)$.

By inspecting the differences between the sources populations with and without the UVW1-magnitude offsets we have found that these sources have different colours. So, it
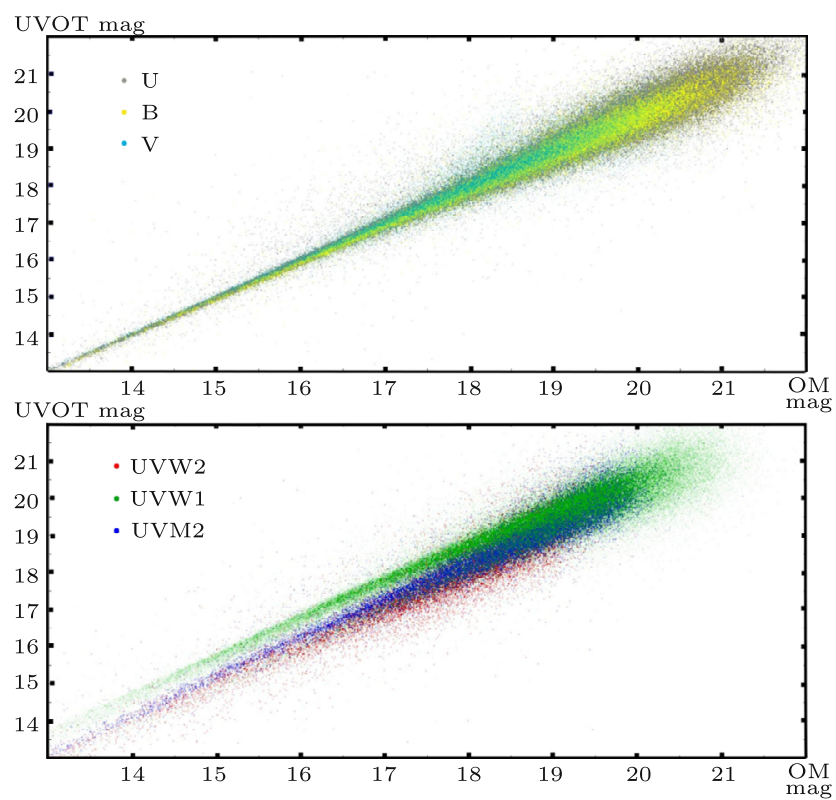

Fig. 4 Comparison between the source magnitudes (Vega) of the OM and UVOT source catalogues for the visible (upper panel) and UV (lower panel) filters

turns out that the UVW1-magnitude offset is not due to a problem with the catalogue data processing, but rather due to a complicated interplay of various factors, such as the difference in the shapes of the UVW1 filters of the OM and UVOT telescopes (Fig. 5), the existence of non-negligible "red-leaks" of these filters and also due to the coincidence losses of source counts in the photodetector. This issue has to be examined in more detail in the future.

The photometric accuracy of the catalogues SUSS-2 and UVOTSSC is characterised by Fig. 6, which shows the dependence of source magnitude errors on source magnitudes (standard deviation) for different OM and UVOT filters. In average, the photometric accuracy of the UVOT catalogue is higher than that of OM due to the use of summed images for source photometry whereas in the case of OM the summed image photometry was made only for part of the sources.

\section{Conclusions}

The ultraviolet source catalogues for $10+$ years of XMM Newton and 5+ years of Swift observations could be used as powerful tools for studying extreme physical processes in compact objects, selecting UV sources for additional followup, finding and studying variable UV sources, characterising dust clouds in different regions and regimes of star formation, detecting dust in stellar winds, determining the fraction of young generation stars in galaxies with high star formation rates, constraining the initial mass function and many other important studies. 

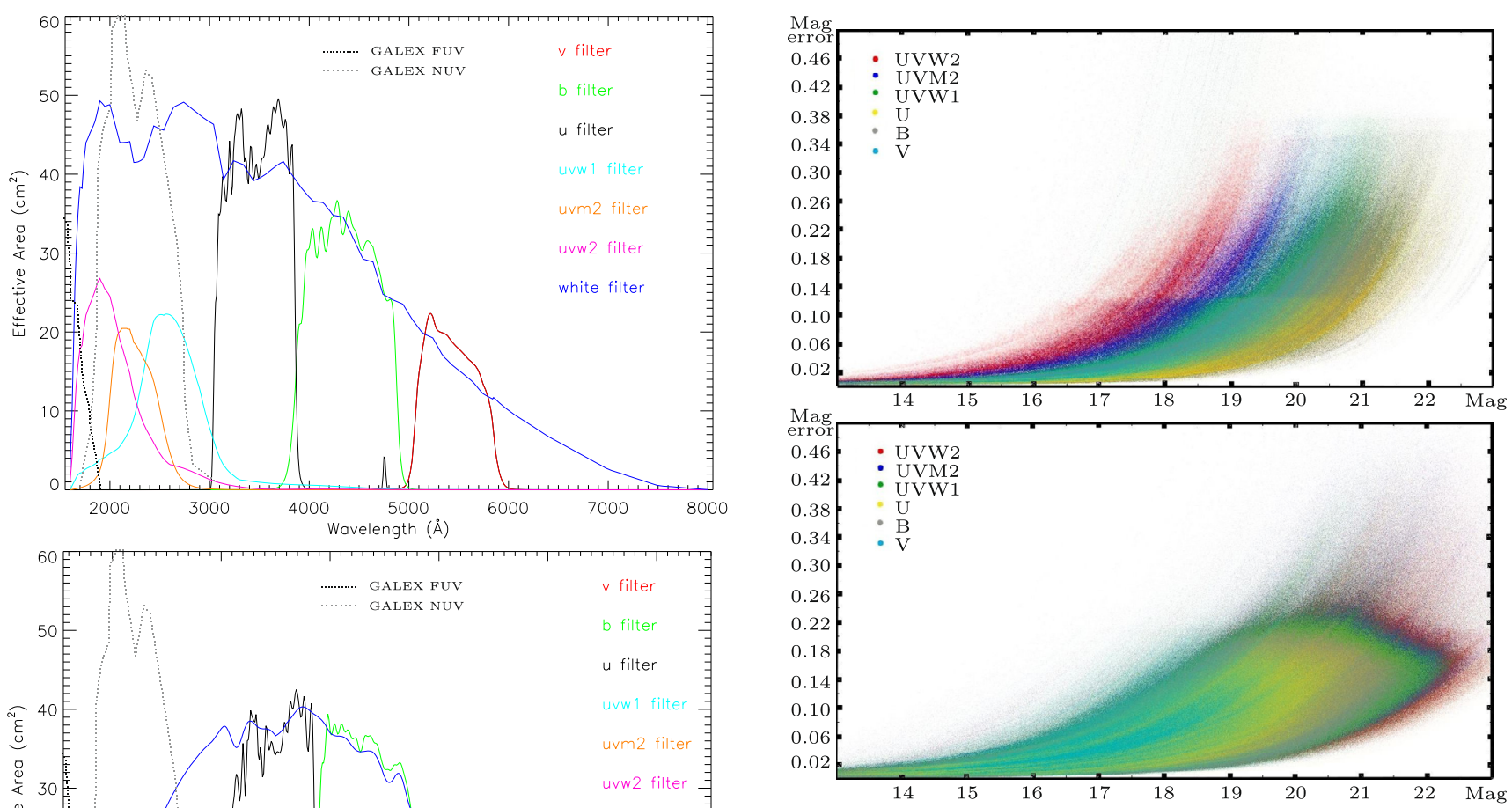

Fig. 6 OM (upper panel) and UVOT (lower panel) magnitude errors as functions of the source (Vega) magnitudes

M. Page, M. Siegel, A. Talavera, A. Breeveld, S. Oates, N.P.M. Kuin, W. Landsman, R. Mignani, M. De Pasquale, P.J. Smith, S. Hunsberger, M. Carter, P.W.A. Roming, M. Cropper, S.T. Holland, F.E. Marshall, M. Chester, P.J. Brown, T.S. Koch, E.A. Hoversten.

Fig. 5 Comparison of the effective areas of the Swift UVOT (above) and XMM OM (below) filters; GALEX FUV and NUV effective areas (dotted lines) are shown as a reference

The catalogues presented here are preliminary. They are expected to be publicly released in March 2014. Currently, they are under the process of verification, validation and correction of possible problems.

Acknowledgements This work is presented on behalf of the Swift UVOT and XMM OM teams whose support is very much appreciated:

Open Access This article is distributed under the terms of the Creative Commons Attribution License which permits any use, distribution, and reproduction in any medium, provided the original author(s) and the source are credited.

\section{References}

Mason, K.O., Breeveld, A., Much, R., et al.: Astron. Astrophys. 365, L27 (2001)

Page, M.J., et al.: Mon. Not. R. Astron. Soc. 426, 903 (2012)

Roming, P., et al.: Space Sci. Rev. 120, 95 (2005) 